\title{
Combined effects of synbiotic and sitagliptin versus sitagliptin alone in patients with nonalcoholic fatty liver disease
}

\author{
Saba Sayari, Hassan Neishaboori, and Maryam Jameshorani \\ Zanjan Metabolic Disease Research Center, Vali-e-Asr Hospital, Zanjan University of Medical Science, Zanjan, Iran
}

Background/Aims: Non-alcoholic fatty liver disease (NAFLD) is one of the most prevalent chronic liver diseases in recent years. The aim of this study was to evaluate the effects of sitagliptin with and without a synbiotic supplement in the treatment of patients with NAFLD.

Methods: In total, 138 NAFLD patients aged 18-60 years were enrolled in the study. Patients were randomized to one of the following treatments for 16 weeks: Group I ( $n=68)$, sitagliptin $50 \mathrm{mg}$ daily plus placebo (one capsule per day) or group II ( $n=70$ ) sitagliptin $50 \mathrm{mg}$ daily plus synbiotic (one capsule per day). Changes in fasting blood glucose (FBS), liver enzymes, lipid profile, and body mass index were compared between the groups.

Results: The mean change in FBS with sitagliptin-placebo from baseline was $-10.47 \pm 5.77 \mathrm{mg} / \mathrm{dL}$, and that with sitagliptin-synbiotic was $-13.52 \pm 4.16 \mathrm{mg} / \mathrm{dL}$. There was a significant difference between the groups $(P<0.001)$. The mean change in cholesterol (Chol) was $-8.34 \pm 28.83 \mathrm{mg} / \mathrm{dL}$ with sitagliptin-placebo and $-21.25 \pm 15.50 \mathrm{mg} / \mathrm{dL}$ with sitagliptinsynbiotic. There was a significant difference between the two groups $(P=0.029)$. The administration of sitagliptin-placebo induced an increase of $6.13 \pm 27.04 \mathrm{mg} / \mathrm{dL}$ in low density lipoprotein (LDL), whereas sitagliptin-synbiotic induced a decrease of $14.92 \pm 15.85 \mathrm{mg} / \mathrm{dL}$ in LDL. A significant difference was observed between the two groups $(P<0.001)$. On the other hand, in the sitagliptin-synbiotic group, there was significant improvement in aspartate aminotransferase (AST) level compared to the sitagliptin-placebo group $(P=0.018)$.

Conclusions: Sitagliptin-synbiotic produced greater improvement in FBS, AST, Chol, and LDL compared to sitagliptin alone in patients with NAFLD. (Clin Mol Hepatol 2018;24:331-338)

Keywords: Non-alcoholic fatty liver disease; Randomized controlled trial; Synbiotic; Sitagliptin

\footnotetext{
Study Highlights
}

This study examined the effects of adding synbiotic to sitagliptin in comparison with sitagliptin alone in patients with non-alcoholic fatty liver disease. It was concluded that the combination of sitagliptin-synbiotic has a better effect on the parameters of fasting blood glucose, aspartate aminotransferase, cholesterol, and low density lipoprotein.

\footnotetext{
Abbreviations:

ALT, alanine aminotransferase; AST, aspartate aminotransferase; BMI, body mass index; CFU, colony forming unit; Chol, cholesterol; DPP-4, dipeptidyl peptidase-4; FBS, fasting blood glucose; GIP, gastric inhibitory polypeptide; GLP, glucagonlike peptide; GLP-1, glucagon-like peptide-1; HCC, hepatocellular carcinoma; HDL, high density lipoprotein; LDL, low density lipoprotein; NAFLD, non-alcoholic fatty liver disease; NASH, non-alcoholic steatohepatitis; TG, triglyceride; ZMDRC, Zanjan Metabolic Diseases Research Center
}

Corresponding author : Saba Sayari

Department of Internal Medicine, Vali-e-Asr Hospital, Zanjan University of Medical Science, Zanjan 45157-77978, Iran

Tel: +98-2433770801, Fax: +98-2433770751

E-mail: sabasayari23@gmail.com

https://orcid.org/0000-0002-7929-128X 


\section{INTRODUCTION}

Non-alcoholic fatty liver disease (NAFLD) is one of the most prevalent chronic liver diseases in the world. ${ }^{1,2}$ NAFLD refers to liver steatosis in the absence of other secondary causes of accumulation of fat in the liver. This chronic disease can be limited to simple steatosis, however, when the disease is associated with inflammation, it can progress to non-alcoholic steatohepatitis (NASH). The likelihood of progression of NASH to liver fibrosis, cirrhosis and eventually to hepatocellular carcinoma (HCC) is high. ${ }^{3-6}$ The possibility of progression of simple steatosis to NASH, chirosis and HCC within a period of 10 years is $12-40 \%, 15-25 \%$, and $7 \%$, respectively.

The prevalence of NAFLD is not precisely obvious, due to the absence of simple and non-invasive ways of diagnosing a large number of patients without any complication. ${ }^{8,9} \mathrm{It}$ is estimated that the worldwide prevalence rate of NAFLD is about $25-45 \%$. $^{10}$

The most significant histological finding in non-alcoholic fatty liver disease is the increase in the level of fat in the liver cells. However, NAFLD pathogenesis is not well known presently, but the most familiar pathway is insulin resistance. On the other hand, oxidative stress and inordinate activation of the immune and inflammatory systems of the body are regarded as another pathway in the pathogenesis of NAFLD. ${ }^{11-13}$ It seems that the successful control of these main pathways involved in the pathogenesis of NAFLD (insulin resistance and oxidative stress with excessive immune and inflammatory activity), is an imperative step in the treatment of NAFLD. Other non-drug therapies that can be adopted in the treatment of NAFLD include lifestyle modification, weight loss and daily physical activity.

Presently, various treatment options that target each aspect of the NAFLD's pathogenesis have been explored and advocated. One of these therapies involves the use of new anti-diabetic drugs that affect the glucagon-like peptide-1 (GLP-1) pathway. This hormone controls blood glucose by stimulating insulin secretion, thereby increasing the oxidation of fatty acids in the liver and the sensitivity of insulin blood glucose levels. These drugs include analogues of GLP-1 (exenatide, liraglutide) and dipeptidyl peptidase-4 inhibitor (sitagliptin, vildagliptin, saxagliptin). DPP-4 is a membrane peptidase that targets GLP-1 and makes it inactive. The inactivation of GLP-1 induces glomerular intolerance and hepatic steatosis. In addition, as regard the activity of peptidase, DPP-4 is associated with stimulation of the immune system, reduction of extracellular matrix and accumulation of lipids. DPP-4 is expressed in high levels in the liver; and by extension, according to information obtained in recent years; DPP-4 has been discovered to be involved in the progression of many chronic liver diseases, such as hepatitis C, NAFLD, and HCC. DPP-4 inhibitors such as sitagliptin, can enhance steatosis, liver damage, and glucose disorders in NAFLD patients; although, they also play a key role in the inhibition and regulation of the immune system involved in the pathogenesis of NAFLD. ${ }^{14,15}$

Additionally, gut dysbiosis is said to be associated with obesity, diabetes, metabolic syndrome and NAFLD through excess energy production, disruption of host energy metabolism, pro-inflammatory signals, and finally, the direct effect of hepatotoxicity of bacterial productions such as ethanol and ammonia. Consequently, changes in the intestinal microbial flora and subsequent inflammatory responses play a significant role in liver diseases, especially NASH and cirrhosis. ${ }^{16-19}$

Prebiotics are indigestible carbohydrates that have beneficial effects on the host by creating a suitable environment for the growth of probiotics. Probiotics, which are also regarded as liver microorganisms have beneficial effects on the host when they are utilized in ample quantity by modulating the intestinal microbial flora and regulating the immune system. Today, there are drugs in the market called synbiotic that combine the properties of prebiotics and probiotics. Daily use of prebiotics and probiotics is believed to slow down the progression of NAFLD and forestall further degeneration of the disease owing to their ability to control intestinal flora, intestinal permeability and inflammatory responses. ${ }^{6,9}$ It therefore seems that the administration of synbiotics in combination with sitagliptin can be more efficacious in the treatment and management of NAFLD patients considering the potency of their complementary roles. The hypothesis we wished to test was whether sitagliptin with and without a synbiotic supplement would be beneficial in the treatment and management of NAFLD patients.

\section{MATERIALS AND METHODS}

Patients with NAFLD were recruited for the randomized, double blind trial between September 20, 2015 and August 15, 2017 from the Vali-e-Asr Hospital in Zanjan, Iran. The study was approved by the Ethics Committee of Zanjan Metabolic Diseases Research Center (ZMDRC), Zanjan University of Medical Sciences (approval number: A-12-500-13).

\section{Subjects}

NAFLD patients between the ages of 18 and 60 years, with body mass index (BMI) ranging from 25 to $29.9 \mathrm{~kg} / \mathrm{m}^{2}$ and who had im- 
paired fasting blood glucose (FBS) (100-125 mg/dL) and/or impaired oral glucose tolerance test (140-199 mg/dL), were screened initially by physical examination and study of their medical history. All patients provided written informed consent to participate in the study. In our study, all the patients were new cases of NAFLD. The diagnosis of NAFLD was made on the basis of the presence of steatosis, on ultrasound examination, associated with elevated alanine aminotransferase (ALT) concentration 1.5-3 times higher than normal upper limit according to sex and age. Those with any history of alcohol consumption more than 10 grams per day in women and more than 20 grams per day in men, respectively were excluded from the study. Exclusion of other liver diseases was made by detailed investigations including hepatitis B and C serology (measured by ELISA with Stat Fax Elisa Reader ${ }^{\circledR}$ ), positive antinuclear antibodies or antimitochondrial antibodies (measured by ELISA with Stat Fax Elisa Reader ${ }^{\circledR}$; Awareness Technology Inc., Palm City, FL, USA), iron profile studies (measured by Kontron GABA Counter ${ }^{\circledR}$; Roche Bioelectronique Kontron, Paris, France), total iron-binding capacity (measured by BT-3500 AutoAnalyzer ${ }^{\circledR}$; Biotecnica Instruments, Via Licenza, Rome, Italy), transferrin saturation (measured by BT3500 AutoAnalyzer $^{\circledR}$ ) and positive ceroplasmin (measured by Elisa with Stat Fax Elisa Reader ${ }^{\circledR}$ ). Other exclusion criteria included presence of kidney disease, thyroid disorders, immunodeficiency diseases, receiving cholesterol lowering medication, current or previous heart failure and being pregnant or breastfeeding.

\section{Study design}

The sample size was computed based on primary information obtained from the study conducted by Wong et al., on ALT. ${ }^{20}$ Considering $\alpha=0.05$ and a power of $80 \%$, the sample size was computed as $66.17(\approx 67)$ per group. This number was increased to 70 per group to accommodate the anticipated dropout rate.

Participants were randomly selected into 2 groups using a block randomization technique of size 4 .

Patients were assigned in a 1:1 ratio to one of the 2 groups for 16 weeks of treatment, either (1) sitagliptin (50 mg once daily) and placebo (maltodexterin) (once daily), or (2) sitagliptin (50 mg once daily) and synbiotic (500 mg once daily).

At enrollment, the NAFLD patients were advised by a dietitian on the need for energy balanced diet and physical activity according to the "Clinical Guidelines on the Identification, Evaluation, and Treatment of Overweight and Obesity in Adults" from the NIH and the North American Association for the Study of Obesity. ${ }^{21}$

In group 1, the treatment commenced with one tablet of sitagliptin per day (50 mg/daily) and one capsule of placebo (maltodexterin) per day. In group 2, the treatment started with one tablet of sitagliptin per day (50 mg/daily) and one capsule of synbiotic per day (500 mg/daily). Each synbiotic capsule (Familakt) contained $10^{9}$ colony forming unit (CFU) of 7 strains of friendly bacteria (Lactobacillus casei, Lactobacillus rhamnosus, Lactobacillus acidophilus, Lactobacillus bulgaricus, Bifidobacterium breve, Bifidobacterium longum, Streptococcus thermophilus) and prebiotic (fructooligosaccharide) and probiotic cultures (magnesium stearate [source: mineral and vegetable] and a vegetable capsule [hydroxypropyl methylcellulose]).

\section{Paraclinical assessments}

The weight and height (anthropometric measurements) of all the patients included in the study were measured at baseline. The weight of the patients was remeasured at week 16. Each individual's BMI before and after treatment was computed using the following formula: BMI=weight (in $\mathrm{kg}$ )/height (in $\mathrm{m}^{2}$ ). In this study, a precision weighing scale with a precision of $0.5 \mathrm{~kg}$, and which was calibrated everyday was employed. To measure the height, the tape meter with a precision of $0.5 \mathrm{~cm}$ was used.

\section{Clinical assessments}

Serum levels of FBS, ALT, aspartate aminotransferase (AST), cholesterol (Chol), triglyceride (TG), low density lipoprotein (LDL) and high density lipoprotein (HDL) were measured at 0 and 16 weeks. All biochemical assessments were carried out in the same laboratory (central laboratory of Vali-e-Asr Hospital) employing standard laboratory techniques. The biochemical assessments were performed on blood collected after overnight fasting (at least 12 hours).

\section{Adherence to treatment protocol}

To ensure that patients adhere to diet and medication regimen, each week a trained person reminds the patients of the necessity and importance of adherence to their treatment regimen through telephone calls.

At the beginning of the study, each patient was informed about the possible side effects of the medications, and advised not to dismiss the researcher and report to the hospital as soon as possible if they had any complications. The phone number of one of the researchers was also made available to patients. 


\section{Statistical analysis}

Data were analyzed employing SPSS software, version 24 (IBM Corp., Armonk, NY, USA) and results were expressed as mean \pm standard deviation or percentage. For all analyses, a $P$-value $<0.05$ was considered statistically significant. The normality of the distribution of variables was investigated by the Kolmogorov-Smirnov test. Chi-square test was used to compare the qualitative variables. Independent $t$-test was employed to compare the median of quantitative variables between the two groups. Paired $t$-test was used to compare the median of quantitative variables before and after treatment in each group. Logistic analysis was used to identify any difference between the two groups after intervention, in terms of lipid profile.

\section{RESULTS}

From September 2015 to August 2017, 140 patients were enrolled in the study and randomly assigned into two groups: sitagliptin-placebo group (group 1) ( $n=70)$ and sitagliptin-synbiotic group (group 2) ( $n=70)$. Sixty-eight patients in the first group and all the patients in the second group completed the study. Patient screening, enrollment, and retention in treatment group are presented in Fig. 1.

In this study, $39.5 \%$ of the participants were female and $60.5 \%$ were male. In the first group, $44.1 \%$ were female and $55.9 \%$ were male while in the second group, $35.7 \%$ were female and $64.3 \%$ were male. There was no significant difference between the two groups $(P$-value $=0.338)$. The baseline clinical and demographic data of the two groups are summarized in Table 1. As shown in the table, there was no significant difference between the two treatment groups in terms of basic characteristics.

At the end of the 16-week treatment period, a significant improvement in liver enzymes was observed in both groups before and after treatment. In comparison with the sitagliptin-placebo group, patients taking sitagliptin-synbiotic had a significantly higher decrease in AST: we reported the amounts of ALT and AST before and after intervention seperately. ALT $(71.60 \pm 21.27$ to $46.42 \pm 17.39$ in the sitagliptin-placebo group and $72.00 \pm 15.60$ to $48.71 \pm 13.65$ in the sitagliptin-synbiotic group; $P<0.456)$, and AST (51.59 \pm 26.89 to $37.30 \pm 18.82$ in the sitagliptin-placebo group and $54.15 \pm 9.89$ to $35.00 \pm 7.89$ in the sitagliptin-synbiotic group; $P<0.018$ ) (Table 2-4).

The two treatment groups had comparable Chol, TG, LDL and HDL at baseline (Table 1). The mean Chol, TG, LDL and HDL levels

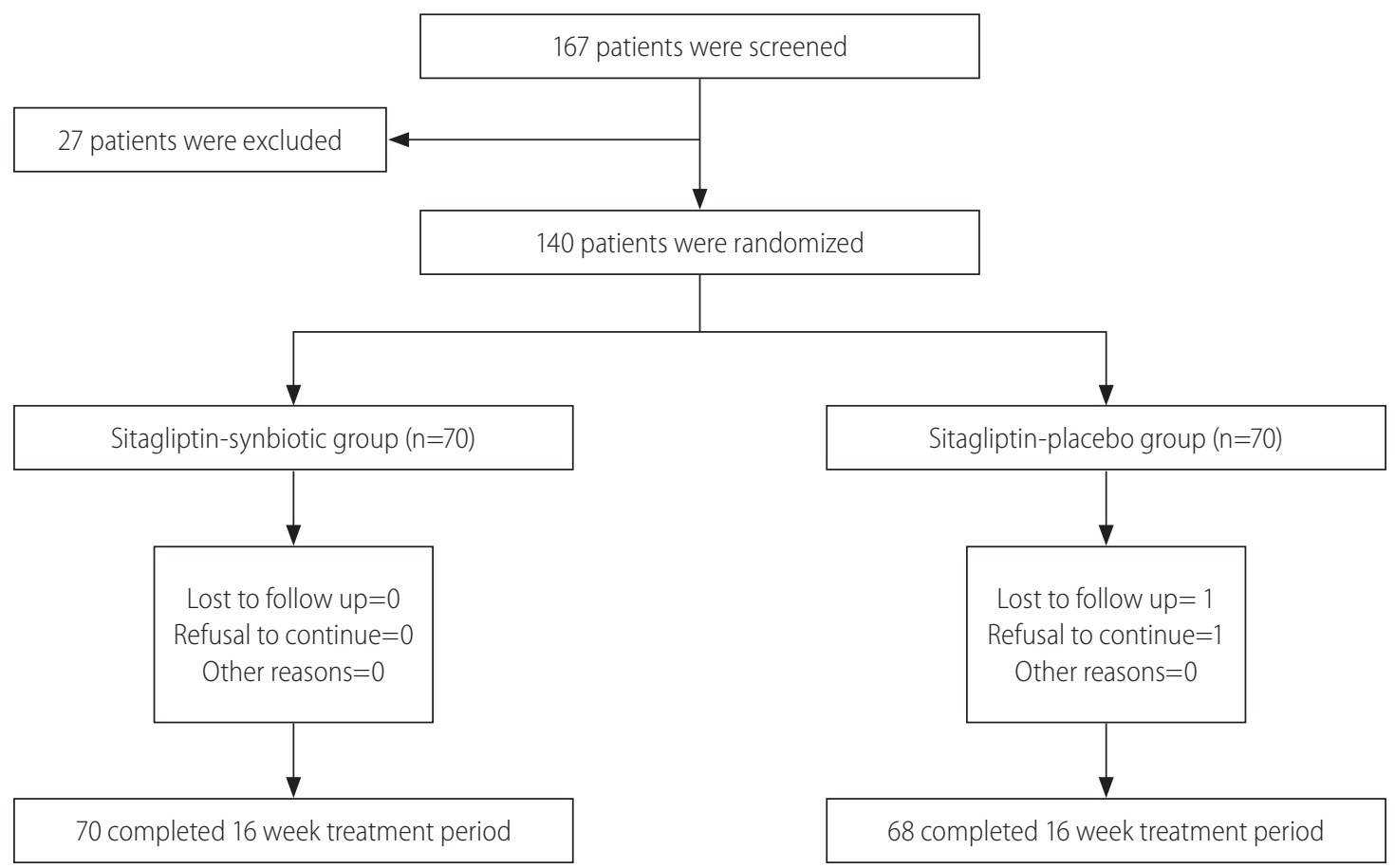

Figure 1. Study flowchart. Flowchart diagram of randomized controlled trials evaluating the use of synbiotics agents in addition to sitagliptin in patients with non-alcoholic fatty liver disease. 
Table 1. Baseline parameters of the patients in each group

\begin{tabular}{lccc}
\hline Variable & Group 1 & Group 2 & P-value \\
\hline Age (year) & $43.42 \pm 11.65$ & $42.48 \pm 11.41$ & 0.669 \\
\hline FBS (mg/dL) & $103.32 \pm 6.23$ & $103.25 \pm 3.63$ & 0.941 \\
\hline ALT (U/L) & $71.60 \pm 21.27$ & $72.00 \pm 15.60$ & 0.906 \\
\hline AST (U/L) & $51.59 \pm 26.89$ & $54.15 \pm 9.89$ & 0.460 \\
\hline Chol (mg/dL) & $197.72 \pm 35.91$ & $205.84 \pm 29.29$ & 0.244 \\
\hline TG (mg/dL) & $229.20 \pm 116.03$ & $203.84 \pm 47.40$ & 0.124 \\
\hline LDL (mg/dL) & $129.48 \pm 28.74$ & $122.45 \pm 25.93$ & 0.237 \\
\hline HDL (mg/dL) & $39.55 \pm 4.84$ & $39.75 \pm 4.09$ & 0.830 \\
Weight (kg) & $85.05 \pm 15.36$ & $86.68 \pm 14.15$ & 0.533 \\
BMl (kg/m $\left.{ }^{2}\right)$ & $29.54 \pm 3.71$ & $29.72 \pm 3.62$ & 0.779 \\
\hline
\end{tabular}

Values are presented as mean \pm SD unless otherwise indicated.

FBS, fasting blood glucose; ALT, alanine aminotransferase; AST, aspartate aminotransferase; Chol, cholesterol; TG, triglyceride; LDL, low density lipoprotein; HDL, high density lipoprotein; BMI, body mass index.

Table 2. The mean and standard deviation of variables in the study before and after treatment in the group treated with sitagliptin-placebo (group 1)

\begin{tabular}{lccc}
\hline Variable & $\begin{array}{c}\text { Before } \\
\text { treatment }\end{array}$ & $\begin{array}{c}\text { After } \\
\text { treatment }\end{array}$ & P-value \\
\hline FBS $(\mathrm{mg} / \mathrm{dL})$ & $103.32 \pm 6.23$ & $92.84 \pm 7.35$ & $<0.001$ \\
\hline ALT $(\mathrm{U} / \mathrm{L})$ & $71.60 \pm 21.27$ & $46.42 \pm 17.39$ & $<0.001$ \\
\hline AST $(\mathrm{U} / \mathrm{L})$ & $51.59 \pm 26.89$ & $37.30 \pm 18.82$ & $<0.001$ \\
\hline Chol $(\mathrm{mg} / \mathrm{dL})$ & $197.72 \pm 35.91$ & $189.37 \pm 33.70$ & 0.130 \\
\hline $\mathrm{TG}(\mathrm{mg} / \mathrm{dL})$ & $229.20 \pm 116.03$ & $194.10 \pm 69.07$ & 0.002 \\
\hline LDL $(\mathrm{mg} / \mathrm{dL})$ & $129.48 \pm 28.74$ & $135.62 \pm 28.89$ & 0.232 \\
\hline HDL $(\mathrm{mg} / \mathrm{dL})$ & $39.55 \pm 4.84$ & $40.24 \pm 4.18$ & 0.538 \\
\hline Weight $(\mathrm{kg})$ & $85.05 \pm 15.36$ & $81.67 \pm 15.07$ & $<0.001$ \\
\hline BMl $\left(\mathrm{kg} / \mathrm{m}^{2}\right)$ & $29.54 \pm 3.71$ & $28.37 \pm 3.67$ & $<0.001$ \\
\hline
\end{tabular}

Values are presented as mean \pm SD unless otherwise indicated.

FBS, fasting blood glucose; ALT, alanine aminotransferase; AST, aspartate aminotransferase; Chol, cholesterol; TG, triglyceride; LDL, low density lipoprotein; HDL, high density lipoprotein; BMI, body mass index.

in the sitagliptin-placebo group increased from $197.72 \pm 35.91$ to $189.37 \pm 33.70(P=0.13), 229.20 \pm 116.03$ to $194.10 \pm 69.07(P=0.002)$, $129.48 \pm 28.74$ to $135.62 \pm 28.89(P=0.232)$ and $39.55 \pm 4.84$ to $40.24 \pm 4.18(P=0.538)$, respectively (Table 2). In the sitagliptin-synbiotic group, Chol, TG, LDL, and HDL levels changed from 205.84 \pm 29.29 to $184.58 \pm 21.78(P<0.001), 203.84 \pm 47.40$ to $177.41 \pm 21.78$ $(P<0.001), 122.45 \pm 25.93$ to $107.53 \pm 19.85(P<0.001)$ and $39.75 \pm$ 4.09 to $41.58 \pm 2.52(P<0.001)$, respectively (Table 3$)$. The difference between the changes observed in the two groups in terms of Chol and LDL was statistically significant (Table 4).
Table 3. The mean and standard deviation of variables in the study before and after treatment in the group treated with sitagliptin and symbiotic (group 2)

\begin{tabular}{lccc}
\hline Variable & $\begin{array}{c}\text { Before } \\
\text { treatment }\end{array}$ & $\begin{array}{c}\text { After } \\
\text { treatment }\end{array}$ & P-value \\
\hline FBS $(\mathrm{mg} / \mathrm{dL})$ & $103.25 \pm 3.63$ & $89.72 \pm 5.21$ & $<0.001$ \\
\hline ALT $(\mathrm{U} / \mathrm{L})$ & $72.00 \pm 15.60$ & $48.71 \pm 13.65$ & $<0.001$ \\
\hline AST $(\mathrm{U} / \mathrm{L})$ & $54.15 \pm 9.89$ & $35.00 \pm 7.89$ & $<0.001$ \\
\hline Chol $(\mathrm{mg} / \mathrm{dL})$ & $205.84 \pm 29.29$ & $184.58 \pm 21.78$ & $<0.001$ \\
\hline $\mathrm{TG}(\mathrm{mg} / \mathrm{dL})$ & $203.84 \pm 47.40$ & $177.41 \pm 21.78$ & $<0.001$ \\
$\mathrm{LDL}(\mathrm{mg} / \mathrm{dL})$ & $122.45 \pm 25.93$ & $107.53 \pm 19.85$ & $<0.001$ \\
\hline $\mathrm{HDL}(\mathrm{mg} / \mathrm{dL})$ & $39.75 \pm 4.09$ & $41.58 \pm 2.52$ & $<0.001$ \\
Weight $(\mathrm{kg})$ & $86.68 \pm 14.15$ & $83.17 \pm 13.91$ & $<0.001$ \\
\hline BMl $\left(\mathrm{kg} / \mathrm{m}^{2}\right)$ & $29.72 \pm 3.62$ & $28.43 \pm 3.50$ & $<0.001$ \\
\hline
\end{tabular}

Values are presented as mean \pm SD unless otherwise indicated.

FBS, fasting blood glucose; ALT, alanine aminotransferase; AST, aspartate aminotransferase; Chol, cholesterol; TG, triglyceride; LDL, low density lipoprotein; HDL, high density lipoprotein; BMI, body mass index.

Table 4. The mean and standard deviation of changes in baseline variables after intervention in both groups

\begin{tabular}{lccc}
\hline Variable & Group 1 & Group 2 & P-value \\
\hline FBS $(\mathrm{mg} / \mathrm{dL})$ & $-10.47 \pm 5.77$ & $-13.52 \pm 4.16$ & 0.001 \\
ALT $(\mathrm{U} / \mathrm{L})$ & $-25.18 \pm 19.11$ & $-23.28 \pm 7.78$ & 0.456 \\
AST $(\mathrm{U} / \mathrm{L})$ & $-14.28 \pm 15.11$ & $-19.15 \pm 7.03$ & 0.018 \\
\hline Chol $(\mathrm{mg} / \mathrm{dL})$ & $-8.34 \pm 28.83$ & $-21.25 \pm 15.50$ & 0.029 \\
\hline TG $(\mathrm{mg} / \mathrm{dL})$ & $-35.10 \pm 56.27$ & $-26.42 \pm 20.83$ & 0.425 \\
LDL $(\mathrm{mg} / \mathrm{dL})$ & $6.13 \pm 27.04$ & $-14.92 \pm 15.85$ & $<0.001$ \\
HDL $(\mathrm{mg} / \mathrm{dL})$ & $0.68 \pm 5.95$ & $1.8 \pm 3.04$ & 0.335 \\
Weight $(\mathrm{kg})$ & $-3.38 \pm 1.97$ & $-3.51 \pm 1.36$ & 0.664 \\
\hline BMI $\left(\mathrm{kg} / \mathrm{m}^{2}\right)$ & $-1.17 \pm 0.70$ & $-1.28 \pm 1.53$ & 0.594 \\
\hline
\end{tabular}

Values are presented as mean \pm SD unless otherwise indicated.

FBS, fasting blood glucose; ALT, alanine aminotransferase; AST, aspartate aminotransferase; Chol, cholesterol; TG, triglyceride; LDL, low density lipoprotein; HDL, high density lipoprotein; BMl, body mass index.

With the use of logistic analysis, it was concluded that the sitagliptin-synbiotic combination therapy was more efficacious than the sitagliptin-placebo composition on blood TG levels in the range of 150 to 200. In addition, this combination therapy showed the highest efficacy in the levels of $130 \leq \mathrm{LDL}<160$ when compared to sitagliptin-placebo combination (Table 5).

FBS also decreased significantly in both groups before and after treatment. The reduction in the sitagliptin-placebo group compared to the sitagliptin-synbiotic group was as follows: FBS decreased as much as $-10.47 \pm 5.77$ compared to $-13.52 \pm 4.16(P<0.001)$. 
Table 5. Logistic regression analysis

\begin{tabular}{|c|c|c|c|c|}
\hline \multirow{2}{*}{ Variable } & \multirow{2}{*}{ Wald } & \multicolumn{2}{|c|}{ 95\% confidence interval } & \multirow{2}{*}{$P$-value } \\
\hline & & Lower bound & Upper bound & \\
\hline \multicolumn{5}{|l|}{ Logistic regression } \\
\hline Abnormal HDL & 0.584 & 0.102 & 2.725 & 0.445 \\
\hline \multicolumn{5}{|l|}{ Multinomial regression } \\
\hline $150 \leq T G<200$ in comparison with $T G<150$ & 8.463 & 0.001 & 0.252 & 0.004 \\
\hline $\mathrm{TG} \geq 200$ in comparison with $\mathrm{TG}<150$ & 1.580 & 0.005 & 0.141 & 0.209 \\
\hline $130 \leq \mathrm{LDL}<160$ in comparison with $\mathrm{LDL}<130$ & 6.615 & 1.496 & 19.754 & 0.010 \\
\hline $\mathrm{LDL} \geq 160$ in comparison with $\mathrm{LDL}<130$ & - & - & - & - \\
\hline
\end{tabular}

HDL, high density lipoprotein; TG, triglyceride; LDL, low density lipoprotein.

Additionally, a significant decrease in weight and BMI was observed in both groups (Table 2 and 3); however, no significant difference was observed between the two groups in relation to the aforementioned variables (Table 4).

Adverse events such as peripheral edema, decreased blood sugar, diarrhea, constipation, nausea, spinal osteoarthritis, upper respiratory tract infection occurred in none of the patients in each treatment groups.

\section{DISCUSSION}

Today, various therapies for the treatment of NAFLD have been proposed; however, the relative risk benefits of the different therapies remain one of the most challenging aspects of the treatment and management of NAFLD. Lifestyle changes including dietary habits and participation in regular physical activity are typically part of the standard recommendations. 22,23

To the best our knowledge, this was the first randomized, double-blind clinical trial that evaluated the effects of combination of sitagliptin-synbiotic in comparison with sitagliptin alone on the characteristics of NAFLD.

The present study revealed that the combination of sitagliptinsynbiotic as against sitagliptin alone significantly reduced the levels of FBS in NAFLD patients. Various studies have suggested that Lactobacillus and Bifidobacteria found in synbiotic and probiotic compounds can enhance the levels of FBS. ${ }^{24,25}$ The reason for this is that the bacteria present in the synbiotic compounds increase glucose uptake by stimulating the production of insulinotropic polypeptides and glucagon-like peptides (GLPs) by modifying the intestinal microbial flora. ${ }^{26}$ On the other hand, sitagliptin is a drug that increases the survival of GLPs especially GLP-1 by inhibiting the enzyme
DPP-4. ${ }^{27-29}$ Consequently, these two drugs can further improve blood glucose levels in patients with fatty liver and impaired fasting plasma glucose by reinforcing each other's effects.

To date, only few studies have been conducted on the effects of sitagliptin on lipid profiles. Our study revealed that treatment with sitagliptin for 16 weeks significantly reduced TG levels in the first group. In parallel to our study, Koren et al. ${ }^{30}$, in their research on patients with type 2 diabetes mellitus reported that treatment with sitagliptin for 3 months significantly affected the level of TG. However, changes in the total serum Chol, LDL, and HDL levels were not statistically significant. The current study notwithstanding, the effects of sitagliptin on improvement of LDL and Chol have also been reported in some studies. This improvement may be due to strict adherence to diet and exercise regimen and the long duration of the studies. ${ }^{31,32}$ How sitagliptin improves lipid profile is not well known, but it is said that gastric inhibitory polypeptide (GIP) can purify the circulating chylomicrons by stimulating the activity of adipose tissue lipoprotein lipase. ${ }^{33,34}$ On the other hand, GLP-1 can decrease the secretion of triacylglycerol after meal. ${ }^{35}$ Consequently, sitaglipin with its protective effects on GIP and GLP-1 can possibly improve lipids status. Our study demonstrated that when synbiotic is added to sitagliptin, it can enhance lipid profiles further. Recently, some studies on animal models have suggested that the use of synbiotic can lead to improvement in lipids status. $^{36,37}$ In addition, some authors have spoken about the positive effects of synbiotics on metabolic syndrome. ${ }^{38}$ The positive effects of synbiotics on lipid profiles might be justified by the production of short chain fatty acids, carbon disulfide, methyl acetate, assimilation of Chol in the gastrointestinal tract, enzymatic deconjugation of bile acids and alteration of Chol to form coprostanol in the in testine. ${ }^{24,39}$ Logically, the more potent effect of sitagliptin-synbiotic seems to be that the two drugs play complementary roles in the 
body in terms of improving lipid profiles.

Furthermore, this study had several limitations. One of the limitations of this study was the lack of histopathological confirmation, which could not be done on ethical grounds. Another limitation of this study was the patients' follow-up period. Perhaps the time of follow-up was not enough for some long-term effects such as weight loss.

Generally, the findings of this study are relevant due to the fact that the major risk factors of NAFLD such as obesity, type 2 diabetes, dyslipidemia, and metabolic syndrome are on the increase owing to the industrialization of societies, the mechanization of a lot of daily activities, and lack of exercise and inappropriate diet. ${ }^{40}$ The prevalence of NAFLD in the coming decades seems to be much higher than its current status. Additionally, NAFLD patients have elevated blood glucose, lipids, blood pressure, and cardiovascular diseases and life expectancy also decreases in these patients. ${ }^{16,41,42}$ Considering the complications, morbidity and mortality resulting from it, it is imperative to pay special attention to the disease. On the other hand, although today, NAFLD's non-pharmacological treatments such as lifestyle modifications, proper diet and increased daily activities are said to be much more efficacious than pharmaceutical treatments; however, due to the failure of patients to adhere to these methods in the long-term, attention to and review of drug therapies is inevitable. ${ }^{17}$

Our results show that the combination of sitagliptin-synbiotic as against sitagliptin alone can better improve AST, FBS, Chol, and LDL in NAFLD patients. The benefits reported with administration of synbiotic in this study support the results of larger studies with histological endpoint in patients with NAFLD. Through this method, a reference for choosing the best options for treatment of NAFLD patients can be provided.

\section{Author contributions}

Saba Sayari conceived the study, analyzed data, and wrote the manuscript. Hassan Neishaboori and Maryam Jameshorani performed diagnosis, selected patients and collected samples.

\section{Acknowledgments}

We appreciate all Vali-e-Asr Hospital personnel.

\section{Conflicts of Interest}

The authors declare that there is no conflict of interest regarding the publication of this paper.

\section{REFERENCES}

1. Vuppalanchi R, Chalasani N. Nonalcoholic fatty liver disease and nonalcoholic steatohepatitis: Selected practical issues in their evaluation and management. Hepatology 2009;49:306-317.

2. Watanabe S, Yaginuma R, Ikejima K, Miyazaki A. Liver diseases and metabolic syndrome. J Gastroenterol 2008;43:509-518.

3. Jou J, Choi SS, Diehl AM. Mechanisms of disease progression in nonalcoholic fatty liver disease. Semin Liver Dis 2008;28:370-379.

4. Harrison SA, Torgerson S, Hayashi PH. The natural history of nonalcoholic fatty liver disease: a clinical histopathological study. Am J Gastroenterol 2003;98:2042-2047.

5. Adams LA, Lymp JF, St Sauver J, Sanderson SO, Lindor KD, Feldstein $A$, et al. The natural history of nonalcoholic fatty liver disease: a population-based cohort study. Gastroenterology 2005;129:113121.

6. Ekstedt M, Franzén LE, Mathiesen UL, Thorelius L, Holmqvist M, Bodemar $G$, et al. Long-term follow-up of patients with NAFLD and elevated liver enzymes. Hepatology 2006;44:865-873.

7. Baffy G, Brunt EM, Caldwell SH. Hepatocellular carcinoma in non-alcoholic fatty liver disease: an emerging menace. J Hepatol 2012;56:1384-1391.

8. Kim D, Kim WR, Kim HJ, Therneau TM. Association between noninvasive fibrosis markers and mortality among adults with nonalcoholic fatty liver disease in the United States. Hepatology 2013;57:13571365.

9. Buss C, Valle-Tovo C, Miozzo S, Alves de Mattos A. Probiotics and synbiotics may improve liver aminotransferases levels in nonalcoholic fatty liver disease patients. Ann Hepatol 2014;13:482-488.

10. Rinella ME. Nonalcoholic fatty liver disease: a systematic review. JAMA 2015:313:2263-2273.

11. Bhatt HB, Smith RJ. Fatty liver disease in diabetes mellitus. Hepatobiliary Surg Nutr 2015;4:101-108.

12. Saponaro C, Gaggini M, Gastaldelli A. Nonalcoholic fatty liver disease and type 2 diabetes: common pathophysiologic mechanisms. Curr Diab Rep 2015;15:607.

13. Jamali R, Jamali A. Non-alcoholic fatty liver disease. KAUMS Journal (Feyz) 2010;14:169-179.

14. Ohki T, Isogawa A, Iwamoto $M$, Ohsugi M, Yoshida $H$, Toda N, et al. The effectiveness of liraglutide in nonalcoholic fatty liver disease patients with type 2 diabetes mellitus compared to sitagliptin and pioglitazone. Scientific World Journal 2012;2012:496453.

15. Yilmaz Y, Yonal O, Deyneli O, Celikel CA, Kalayci C, Duman DG. Effects of sitagliptin in diabetic patients with nonalcoholic steatohepatitis. Acta Gastroenterol Belg 2012;75:240-244.

16. Shavakhi A, Minakari M, Firouzian $H$, Assali R, Hekmatdoost $A$, Ferns $G$. Effect of a probiotic and metformin on liver aminotransferases in non-alcoholic steatohepatitis: a double blind randomized 
clinical trial. Int J Prev Med 2013;4:531-537.

17. Younossi ZM, Stepanova M, Afendy M, Fang Y, Younossi Y, Mir $H$, et al. Changes in the prevalence of the most common causes of chronic liver diseases in the United States from 1988 to 2008. Clin Gastroenterol Hepatol 2011;9:524-530.e1; quiz e60.

18. Targher G, Day CP, Bonora E. Risk of cardiovascular disease in patients with nonalcoholic fatty liver disease. N Engl J Med 2010;363:1341-1350.

19. Targher G, Marra F, Marchesini G. Increased risk of cardiovascular disease in non-alcoholic fatty liver disease: causal effect or epiphenomenon? Diabetologia 2008;51:1947-1953.

20. Wong VW, Won GL, Chim AM, Chu WC, Yeung DK, Li KC, et al. Treatment of nonalcoholic steatohepatitis with probiotics. A proofof-concept study. Ann Hepatol 2013;12:256-262.

21. National Institutes of Health, National Heart, Lung, and Blood Institute North American Association for the Study of Obesity. The Practical Guide: Identification, evaluation, and treatment of overweight and obesity in adults. Bethesda (MD): National Institutes of Health, National Heart, Lung, and Blood Institute, NHLBI Obesity Education Initiative, North American Association for the Study of Obesity, 2000.

22. Lonardo A, Targher G. NAFLD: is there anything new under the sun? Int J Mol Sci 2017;18. pii: E1955.

23. Barb D, Portillo-Sanchez P, Cusi K. Pharmacological management of nonalcoholic fatty liver disease. Metabolism 2016;65:1183-1195.

24. Shakeri H, Hadaegh H, Abedi F, Tajabadi-Ebrahimi M, Mazroii N, Ghandi $Y$, et al. Consumption of synbiotic bread decreases triacylglycerol and VLDL levels while increasing HDL levels in serum from patients with type-2 diabetes. Lipids 2014;49:695-701.

25. Barengolts E. Gut microbiota, prebiotics, probiotics, and synbiotics in management of obesity and prediabetes: review of randomized controlled trials. Endocr Pract 2016;22:1224-1234.

26. Al-Salami H, Butt G, Fawcett JP, Tucker IG, Golocorbin-Kon S, Mikov $M$. Probiotic treatment reduces blood glucose levels and increases systemic absorption of gliclazide in diabetic rats. Eur J Drug Metab Pharmacokinet 2008;33:101-106.

27. Goh GBB, Dasarathy S, McCullough A. Pharmacologic management of non-alcoholic fatty liver disease. Adv Pharmacoepidemiol Drug Saf 2014;3:157. doi:10.4172/2167-1052.1000157.

28. Olaywi M, Bhatia T, Anand S, Singhal S. Novel anti-diabetic agents in non-alcoholic fatty liver disease: a mini-review. Hepatobiliary Pancreat Dis Int 2013;12:584-588.

29. Hardy T, Anstee QM, Day CP. Nonalcoholic fatty liver disease: new treatments. Curr Opin Gastroenterol 2015;31:175-183.

30. Koren S, Shemesh-Bar L, Tirosh A, Peleg RK, Berman S, Hamad RA, et al. The effect of sitagliptin versus glibenclamide on arterial stiff- ness, blood pressure, lipids, and inflammation in type 2 diabetes mellitus patients. Diabetes Technol Ther 2012;14:561-567.

31. Monami M, Lamanna C, Desideri CM, Mannucci E. DPP-4 inhibitors and lipids: systematic review and meta-analysis. Adv Ther 2012;29:14-25.

32. Duvnjak L, Blaslov K. Dipeptidyl peptidase-4 inhibitors improve arterial stiffness, blood pressure, lipid profile and inflammation parameters in patients with type 2 diabetes mellitus. Diabetol Metab Syndr 2016;8:26.

33. Wasada T, McCorkle K, Harris V, Kawai K, Howard B, Unger RH. Effect of gastric inhibitory polypeptide on plasma levels of chylomicron triglycerides in dogs. J Clin Invest 1981;68:1106-1107.

34. Kim SJ, Nian C, McIntosh CH. Activation of lipoprotein lipase by glucose-dependent insulinotropic polypeptide in adipocytes. A role for a protein kinase $B, L K B 1$, and AMP-activated protein kinase cascade. J Biol Chem 2007;282:8557-8567.

35. Meier JJ, Gethmann A, Götze O, Gallwitz B, Holst JJ, Schmidt WE, et al. Glucagon-like peptide 1 abolishes the postprandial rise in triglyceride concentrations and lowers levels of non-esterified fatty acids in humans. Diabetologia 2006;49:452-458.

36. Liong MT, Dunshea FR, Shah NP. Effects of a synbiotic containing Lactobacillus acidophilus ATCC 4962 on plasma lipid profiles and morphology of erythrocytes in hypercholesterolaemic pigs on highand low-fat diets. Br J Nutr 2007;98:736-744.

37. Roselino MN, Pauly-Silveira ND, Cavallini DC, Celiberto LS, Pinto RA, Vendramini RC, et al. A potential synbiotic product improves the lipid profile of diabetic rats. Lipids Health Dis 2012;11:114.

38. Beserra BT, Fernandes $R$, do Rosario VA, Mocellin MC, Kuntz MG, Trindade EB. A systematic review and meta-analysis of the prebiotics and synbiotics effects on glycaemia, insulin concentrations and lipid parameters in adult patients with overweight or obesity. Clin Nutr 2015;34:845-858.

39. Ooi LG, Liong MT. Cholesterol-lowering effects of probiotics and prebiotics: a review of in vivo and in vitro findings. Int J Mol Sci 2010;11:2499-2522.

40. Lirussi F, Mastropasqua E, Orando S, Orlando R. Probiotics for nonalcoholic fatty liver disease and/or steatohepatitis. Cochrane Database Syst Rev 2007;(1):CD005165.

41. Abenavoli L, Scarpellini E, Rouabhia S, Balsano C, Luzza F. Probiotics in non-alcoholic fatty liver disease: which and when. Ann Hepatol 2013;12:357-363.

42. Eslamparast T, Poustchi $H$, Zamani F, Sharafkhah M, Malekzadeh $R$, Hekmatdoost A. Synbiotic supplementation in nonalcoholic fatty liver disease: a randomized, double-blind, placebo-controlled pilot study. Am J Clin Nutr 2014;99:535-542 